\title{
A simple model for the anomalous intrinsic viscosity of dendrimers
}

\author{
Yuyuan Lu,,$^{a}$ Tongfei Shi, $\dagger^{a}$ Lijia An, ${ }^{* a}$ Liping $\mathrm{Jin}^{b}$ and Zhen-Gang Wang \\ Received 5th March 2010, Accepted 28th April 2010 \\ First published as an Advance Article on the web 14th May 2010 \\ DOI: 10.1039/c0sm00061b
}

The intrinsic viscosity of dendrimers in solution shows several anomalous behaviors that have hitherto not been explained within the existing theoretical frameworks of either Zimm or Rouse. Here we propose a simple two-zone model based on the radial segmental density profile of the dendrimers and combine a non-draining core with a free-draining outer region description, to arrive at a simple formula that captures most of the main features in the intrinsic viscosity data obtained in experiments.

Dendrimers form a new class of polymeric material with promising applications in a wide range of areas: speciality fluids, biomedical materials, nano-materials, drug-delivery, genetic engineering, etc. ${ }^{1-3}$ The richness of their properties has been the subject of extensive theoretical, ${ }^{4-7}$ experimental ${ }^{8,9}$ and simulation studies. ${ }^{10-17}$ Some of the applications of dendrimers rely on their unique rheological behavior. ${ }^{1,2}$ A key property characterizing the rheological behavior of polymer solutions is the intrinsic viscosity, commonly denoted as $[\eta]$. Experimental data by Fréchet and co-workers ${ }^{\mathbf{8}, 9}$ reveal three distinct features in the behavior of $[\eta]$ : (i) with increasing number of generations $G,[\eta]$ passes through a maximum value; (ii) the generation number $G$ for the tridendrons at which $[\eta]$ reaches a maximum is less than that for the monodendrons; (iii) $[\eta]$ of the tridendrons is higher than that of the monodendrons at small $G$, but lower at higher $G$. These anomalous behaviors have hitherto not been explained within existing theoretical frameworks of either Zimm or Rouse.

The classical Mark-Houwink-Sakurada (MHS) equation for linear polymer solutions $\left([\eta]=K M^{\alpha}\right.$, where $K$ and $\alpha$ are specific constants for a polymer-solvent system, and $M$ is the molecular weight) is completely incapable of describing these observations. The power law dependence of $[\eta]$ on the molecular weight $M$ in the MHS equation stems from the power law dependence of the radius of viscosity $R_{\eta}$ on $M$, which clearly does not hold in dendrimers due to the lack of selfsimilarity (scale invariance) between the different generations; ${ }^{18}$ see Fig. 1. Here $R_{\eta}$ is defined as the radius of viscosity of an effective compact sphere according to the Einstein equation for the intrinsic viscosity $\left([\eta]=(10 \pi / 3) R_{\eta}{ }^{3} / M\right){ }^{19}$ The Fox-Flory formula ${ }^{20,21}$ ( $[\eta]=\Phi\left(6^{1 / 2} R_{g}\right)^{3} / M$, where $\Phi=0.425 N_{A v}$ is a universal constant for all polymer-solvent systems, $N_{A v}$ is Avogadro's number, and $R_{g}$ is the radius of gyration) can capture the existence of a maximum in $[\eta]$ but the position of the maximum and the shapes of the $[\eta] v s$. $G$ predicted by the Fox-Flory formula are grossly deviant from experimental

${ }^{a}$ State Key Laboratory of Polymer Physics and Chemistry, Changchun Institute of Applied Chemistry, Chinese Academy of Sciences, Changchun, 130022, People's Republic of China.E-mail: ljan@ciac.jl.cn

${ }^{b}$ College of Physics, Jilin University, Changchun, 130021, P.R. China

${ }^{c}$ Division of Chemistry and Chemical Engineering, California Institute of Technology, Pasadena, CA 91125, USA

$\dagger$ These two authors contributed equally to this work. data, and the formula fails completely to capture the correlation of $[\eta]$ between the tridendrons and monodendrons. The Fox-Flory equation assumes that the radius of viscosity $R_{\eta}$ is proportional to the hydrodynamic radius $R_{h}$, which is in turn assumed to be proportional to the radius of gyration $R_{g}$. Recently Ferla finds that, unlike linear polymers, the proportionality relationship between $R_{g}$ and $R_{\eta}$ or between $R_{h}$ and $R_{\eta}$ does not hold for dendrimers. ${ }^{22}$ Therefore, for dendrimers, $R_{\eta}$ cannot be simply substituted by $R_{g}$ or $R_{h}$. In addition, fitting of the results of scattering experiments ${ }^{23,24}$ based on the Zimm model gives poor agreement and yields a large overestimate for $R_{g}$ for the larger dendrimer generations. The presence of an inner dense core region introduces two important effects. First, solvent molecules will be effectively trapped inside the core, and second, propagation of flow perturbations near the core region will likely be hindered by the
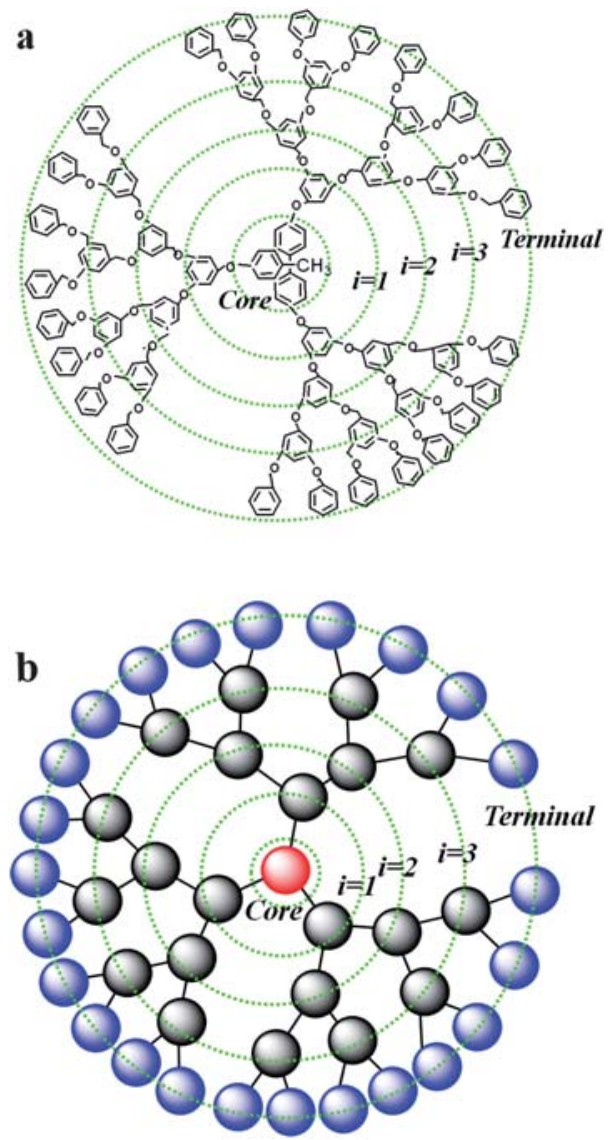

Fig. 1 Schematics of a dendrimer and the coarse grained model. (a) A polybenzylether (PBzE) three-generation tridendron. (b) A coarsegrained model of three-generation tridendron. Note that this structure is termed a four-generation dendrimer in some literature. ${ }^{5}$ 
presence of an effectively solid sphere, thus shielding the hydrodynamic interactions between monomers in the outer region. Treatment of the hydrodynamic interactions using the two-body Oseen tensor or the improved Rotne-Prager tensor ${ }^{25}$ are inadequate. To date, although several studies have focused on the first feature (the maximum of intrinsic viscosity $v s . G),{ }^{1,8,12,15}$ there are no theories that can capture all the three features mentioned above, let alone producing agreement with experimental data with acceptable accuracy. ${ }^{26,27}$

In this paper we propose a simple two-zone model for a dendrimer based on examining the radial segmental density profile, which suggests a combined Zimm and Rouse description of the intrinsic viscosity. The result is a simple formula that captures all the three aforementioned features for $[\eta]$ and yields reasonably good quantitative agreement with existing experimental data.

We propose a two-zone model in which the dendrimer is approximated to be consisting of a dense core that is impermeable to the flow field and a thin region in which the solvent drains freely. The model is based on an examination of the density profile $\rho(R, G)$ of a dendrimer, which denotes the distribution of monomers as a function of the distance $(R)$ from the molecular centre of mass. Fig. 2a shows the density profiles for the coarse-grained model of dendrimers (Fig. 1b) calculated using the self-consistent mean field theory with
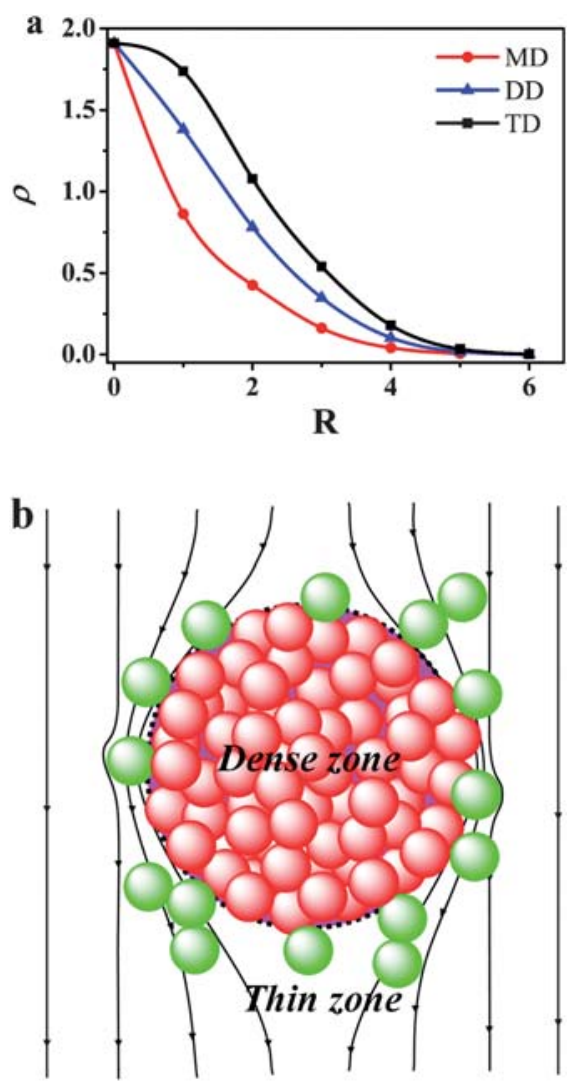

Fig. 2 The two-zone model corresponding to the radial segmental density profiles. (a) The radial segmental density profile of monodendron (MD), didendron (DD) and tridendron (TD). The volume fraction is $\varphi=\rho v$, where $v$ is the excluded volume. (b) A schematic of the two-zone model: the balls inside the dotted circle belong to the dense part and the balls outside the dotted circle belong to the thin part; the distorted lines denote the flow field around the structure. consideration of excluded volume effect. ${ }^{5}$ In these calculations, we assume: (i) the dendrimer is a highly spherically symmetric molecule so that the center of the molecule and the center of mass approximately coincide; (ii) the length of all the successive bonds are equal and are set to 1 in our units; (iii) differences among the monomers of the core, spacers and terminal groups in the dendrimer are ignored (Fig. 1b).

The most relevant feature of the density distribution shown in Fig. $2 \mathrm{a}$ is the high density in the core region - the average volume fraction near the center of the core exceeds 0.5 for our choice of parameters. Such a high density core most certainly makes it difficult for the solvent flow field to penetrate. In other words, solvent molecules in the core region will move with the dendrimer and contributes little to the frictional dissipation as the dendrimer moves in the solvent or vice versa. Also evident in Fig. 2a is the fact that the monomer density drops to very low values beyond some radial distance from the center. In this low density region, although there is still in principle hydrodynamic interactions, these interactions will be weak because (i) the average distance between the non-bonded monomers is large, and (ii) the presence of the dense core shields the long range interactions. The solvent molecules can therefore flow through this region relatively freely, and in our simple two-zone model, we simply take the free draining limit in this region. ${ }^{28} \mathrm{We}$ point out that it is possible to allow a continuously varying degree of drainage by constructing some density dependent permeability function in the spirit of Darcy's law for flow in porous media; $;{ }^{29}$ very similar results to the two-zone model are obtained. As the two-zone model is conceptually and mathematically simpler, we adopt this model in the work presented here. Rathgeber and co-workers ${ }^{30}$ found that a fuzzy sphere model based on a compact sphere with a fuzzy surface can serve as a better description of the SAXS experimental data of all dendrimer generations, lending indirect support to the validity of our two-zone model; the picture of a dense-core was also suggested in ref. 17.

Once we accept the two-zone model, the intrinsic viscosity of the dendrimers can be calculated straightforwardly by noting that the contributions to the viscosity from these two zones are additive. ${ }^{31} \mathrm{We}$ take the dense zone as a solid sphere of radius $R_{c}(G)$ and treat the thin zone as made of free draining Rouse chains with a total number of monomers $N_{1}(G)$, determined from

$$
N_{1}(G)=\int_{R c}^{\infty} \rho(R, G) 4 \pi R^{2} d R
$$

The intrinsic viscosity is then,

$$
[\eta(G)]=\frac{10 \pi\left[R_{c}(G)^{3}+k N_{1}(G) \sigma^{3}\right]}{3 N(G) M_{0}}
$$

where we have used Einstein's intrinsic viscosity formula ${ }^{19}$ for solid spheres. In eqn (2), $M_{0}$ is the mass of a monomer, $\sigma$ is the radius of a monomer and $N(G)$ is the total number of monomers in the dendrimer calculated from:

$$
N(G)=1+j\left(2^{G+1}-1\right)
$$

where $j=1,2,3, \ldots$, for monodendron, didendron, tridendron, etc. Because the $N_{1}(G)$ segments in the thin zone are not isolated but are part of connected chain segments, in order to quantify the effect of correlation, we introduce a coefficient of correlation $k$, which 
represents the ratio between the total effective volume of the segments in the thin zone and their real volume. A first-principles calculation of $k$ is difficult because the "chains" in the thin region do not have well defined lengths or even architectures. In addition, they are connected to the core region. However, we provide an estimate based on the following considerations. The volume of viscosity (as defined through the Einstein equation) for a collection of connected objects is always greater than the sum of the volume of the individual objects; therefore the coefficient $k$ is always greater than 1 . From the density profile, the contribution to the thin region comes primarily from the monomers of the last generation and terminal monomers (cf. Fig. 1b). We have therefore estimated the effective volume associated with a terminal trimer. We take the geometry to be that of an equilateral trimer with center-to-center separation of $b$ and construct a larger sphere centered at the center of the trimer and extending to the center of each of the monomers. The effective volume of the trimer is assumed to be that of the larger sphere plus volumes of the monomer not included in the larger sphere. Using our values for Kuhn length $b$ and $\sigma$, we obtain $k=1.53$. Considering that the trimer is connected to the dense nondraining zone, $k$ will be larger than this value because of the increase of the effective volume of the thin part, and we take it to be 1.7 in our calculations. The same value is used for monodendrons, didendrons and tridendrons. Alternatively, $k$ can be taken as an adjustable parameter in our model to be obtained by best fit to experimental data.

The choice of a cut-off radius $R_{c}(G)$ for the dense core is somewhat arbitrary in the absence of a more fundamental theory. Here we choose $R_{c}(G)$ as a cut-off distance from the density profile, based on the following intuitive consideration. In order for the solvent molecules to drain freely, the average spacing between two monomers has to be at least twice as large as the size of a solvent molecule (assuming the size of a monomer and that of a solvent molecules are the same). If we take the inverse of the cube root of the density as an average measure of the distance between monomers, this implies a density of 0.125 . However, the monomers in the dendrimer are connected. If we consider the average spacing between two strands, this density would correspond to a larger inter-strand spacing. Considering this factor, we increase the threshold density to 0.25 and use this value for all the systems we consider. Thus we choose $R_{c}(G)$ to be the distance from the center of a dendrimer at which the monomer density decreases to $0.25 .^{28}$

Using eqn (2), we have calculated the intrinsic viscosity for the polybenzylether (PBzE) dendrimers studied in ref. 8 and 9 as

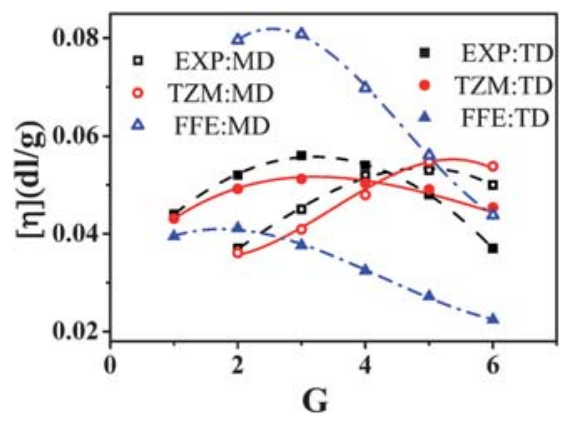

Fig. 3 Comparison of intrinsic viscosity data. The intrinsic viscosity calculated using our two-zone model (TZM), using the Fox-Flory equation (FFE) and from experimental data (EXP) for the PBzE dendrons. ${ }^{8}$ MD: monodendron; TD: tridendron. a function of the generation number $G$. According to experiment and simulation, ${ }^{11-14}$ the excluded volume $v$ is chosen to be 0.3 in our system for the PBzE. The Kuhn length is $1.4 \mathrm{~nm}$, which is taken to be our unit of length. And the molar mass of monomers is $106 \mathrm{~g} \mathrm{~mol}^{-1}$. The comparison is shown in Fig. 3. The results from the calculation agree well with experimental data for the monodendrons and tridendrons. All the three main features about the experimental data alluded to in the opening part of this paper are captured by the calculation. A noticeable discrepancy in Fig. 3 is that the peak value of the intrinsic viscosity for the tridendron is higher than that for the monodendron from the experimental data, whereas the calculation predicts the opposite. Given the simplicity of our model, the overall agreement with experimental data is remarkably good. As a contrast, we show in Fig. 3 the comparison between results obtained using the Fox-Flory formula. Because the intrinsic viscosity predicted by the Fox-Flory theory is far less than the experimental data, we amplify the parameter $\Phi$ by a factor of 1.6 so as to have the calculated and experimental data in the same range. Even allowing the "universal" parameter $\Phi$ to be adjusted, the agreement is very poor. Other than the existence of a peak, whose position is quite a bit off, the FoxFlory formula fails to describe the experimental data.

That solvent molecules in the core region move with the core in flow is an important consideration in our model. Since the ability of the core to drag the solvent molecules within it is relevant for such applications as drug carrier and scavenger for harmful molecules, we introduce a quantitative measure, $\Omega$, defined as the average volume of solvent molecules in the core region per monomer,

$$
\Omega=\frac{V_{s}(G)}{N(G)}=\frac{4 \pi\left[R_{c}(G)^{3}-N_{c}(G) \sigma^{3}\right]}{3 N(G)}
$$

where $V_{s}(G)$ is the total volume of solvents and $N_{c}(G)=N(G)-$ $N_{1}(G)$ is the total number of the monomers, both in the dense core of the dendrimer.

Fig. 4 shows the relationship between $\Omega$ and the generation number $G$. The plot exhibits the same trend as the intrinsic viscosity shown in the inset. In fact, to a large extent, the intrinsic viscosity should reflect the ability of the dendrimer to drag solvent along. According to eqn (4), we see that the maximum value of $\Omega$ arises from the competition between the increase of the volume and the increase of the monomer number density in the dense part with the increase of generation, with the former effect dominating at low generation, and

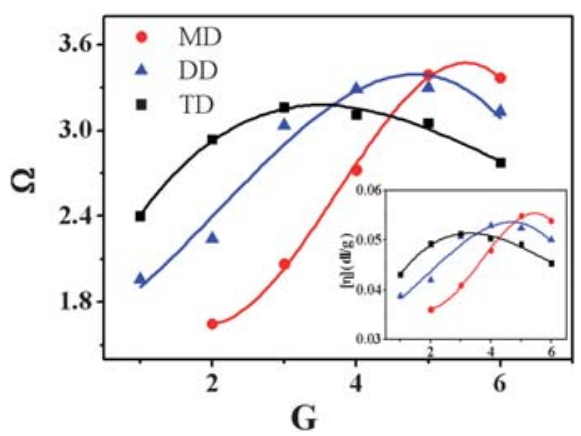

Fig. 4 The average volume of solvent molecules in the core region per monomer as a function of the generation of various dendrimers. Note that the inset shows the intrinsic viscosity calculated using our two-zone model, the unit of the volume is the volume of a monomer, $v=4 \pi \sigma^{3} / 3$ and experimental data for the PBzE didendron (DD) is not available. 
the latter at high generation. As the monomer density is higher for the tridendrons compared to didendrons and monodendrons, the peak in $\Omega$ is reached at lower generation.

We have demonstrated that, by combining a non-draining with a free draining description, our two-zone model based on the radial segmental density profile of the dendrimers captures the key features of the intrinsic viscosity $[\eta]$ for dendrimers and yields reasonably good quantitative agreement with existing experimental data. In addition, we clarify the maximum of $[\eta] v s$. $G$ corresponds to the maximum capacity of dendrimer to drag solvent molecules, an important property for applications as carrier materials and rheology modifiers. More generally our results suggest that the dynamics of dilute polymer solutions may not be adequately described by either the Zimm or Rouse model, but may exhibits combined behaviors of the two models. The dynamics of hyper-branched polymers in solution has already been shown to deviate from the Zimm behavior, because the exponent $\alpha$ in the MHS relationship is much less than 0.5 for randomly branched macromolecules. ${ }^{32}$ And the experimental values of $R_{g} / R_{h}$ are always smaller than the theoretical estimates based on the Zimm model for various polymer structures. ${ }^{20}$ There is clearly a need for a more universal theory to account for the dynamics of polymers with arbitrary architectures. The idea put forward in this work may serve as a stepping stone for developing a more systematic theory for other complex architectures.

\section{Acknowledgements}

This work was supported by the National Natural Science Foundation of China (NSFC) (No. 20734003) and the Fund for Creative Research Groups (No. 50921062), further subsidized by the Special Funds for National Basic Research Program of China (No. 2009CB930100). T. F. S. thanks the support from NSFC (No. 20774096). L. P. J. thanks the support from NSFC (No. 10573007 and No. 10873006).

\section{References}

1 J. M. J. Fréchet and D. A. Tomalia, Dendrimers and Other Dendritic Polymers, Wiley, Chichester, England, 2001, ch. 14.

2 J. M. J. Fréchet, C. J. Hawker, I. Gitsov and J. W. Leon, J. Macromol. Sci., Part A: Pure Appl. Chem., 1996, 33, 1399.

3 F. Vogtle, S. Gestermann, R. Hesse, H. Schwierz and B. Windisch, Prog. Polym. Sci., 2000, 25, 987.
4 P.-G. de Gennes and H. Hervet, J. Phys., Lett., 1983, 44, 351.

5 D. Boris and M. Rubinstein, Macromolecules, 1996, 29, 7251.

6 T. C. Zook and G. T. Pickett, Phys. Rev. Lett., 2003, 90, 015502.

7 B. M. Mladek, G. Kahl and C. N. Likos, Phys. Rev. Lett., 2008, 100, 028301.

8 T. H. Mourey, S. R. Turner, M. Rubinstein, J. M. J. Fréchet, C. J. Hawker and K. L. Wooley, Macromolecules, 1992, 25, 2401.

9 J. M. J. Fréchet, Science, 1994, 263, 1710.

10 M. Ballauff and C. N. Likos, Angew. Chem., Int. Ed., 2004, 43, 2998.

11 M. L. Mansfield and L. I. Klushin, Macromolecules, 1993, 26, 4262.

12 R. L. Lescanec and M. Muthukumar, Macromolecules, 1990, 23, 2280 .

13 M. Murat and G. S. Grest, Macromolecules, 1996, 29, 1278.

14 K. Karatasos, D. B. Adolf and G. R. Davies, J. Chem. Phys., 2001, 115, 5310 .

15 A. V. Lyulin, G. R. Davies and D. B. Adolf, Macromolecules, 2000, 33, 3294 .

16 G. D. R. Echenique, R. R. Schmidt, J. J. Freire, J. G. H. Cifre and J. G. de Torre, J. Am. Chem. Soc., 2009, 131, 8548.

17 H. M. Harreis, C. N. Likos and M. Ballauff, J. Chem. Phys., 2003, 118, 1979.

18 I. B. Rietveld and J. A. M. Smit, Macromolecules, 1999, 32, 4608.

19 A. Einstein, Ann. Phys., 1911, 339, 591.

20 M. Rubinstein and R. H. Colby, Polymer Physics, Ch. 8. Oxford Univ, 2004.

21 M. Doi and S. F. Edwards, The Theory of Polymer Dynamics, Clarendon, 1989, ch. 4.

22 R. L. Ferla, J. Chem. Phys., 1997, 106, 688.

23 T. J. Prosa, B. J. Bauer, E. J. Amis, D. A. Tomalia and R. Scherrenberg, J. Polym. Sci., Part B: Polym. Phys., 1997, 35, 2913.

24 T. J. Prosa, B. J. Bauer and E. J. Amis, Macromolecules, 2001, 34, 4897.

25 J. Rotne and S. Prager, J. Chem. Phys., 1969, 50, 4831.

26 Z. Y. Chen and C. Z. Cai, Macromolecules, 1999, 32, 5423.

27 J. J. Freire, Soft Matter, 2008, 4, 2139.

28 Note this assumption does not contradict with the fact that hydrodynamic interactions persist for long linear polymer coils in dilute solutions because the pervaded volume of a long linear polymer coil is large and the segments are strongly correlated in the pervaded volume. Here we can ignore the hydrodynamic interactions because the segments in the thin part of the dendrimer are very short, consisting of just a few monomers, and are largely uncorrelated, emitting from different parts of the dense core.

29 A. E. Scheidegger, The Physics of Flow Through Porous Media, Toronto Univ., 1974, ch. 6.

30 S. Rathgeber, M. Monkenbusch, M. Kreitschmann, V. Urban and A. Brulet, J. Chem. Phys., 2002, 117, 4047.

31 According to Einstein's equation, independent entities contribute to the viscosity independently. We have effectively assumed that the core and the outer regions contribute independently. The dense core, being approximated as a solid sphere of some effective radius, is largely unaffected by the outer region, whereas the parameter $k$ partially accounts for the coupling of the outer region to the core. 32 W. Burchard, Adv. Polym. Sci., 1999, 143, 113. 